Página inicial: 241 - Página final: 256

TIPO DE ARTÍCULO: de Investigación

\title{
PREOCUPACIÓN AMBIENTAL ENTRE POBLACIÓN UNIVERSITARIA: REPRESENTACIONES SOCIALES E IMPLICACIÓN PERSONAL EN TEMAS AMBIENTALES EN LA UNIVERSIDAD DE ANTIOQUIA.
}

\begin{abstract}
ENVIRONMENTAL CONCERN AMONG UNIVERSITY POPULATION: SOCIAL REPRESENTATIONS AND PERSONAL INVOLVEMENT IN ENVIRONMENTAL ISSUES AT UNIVERSITY OF ANTIOQUIA.
\end{abstract}

\author{
Recibido: Junio 2013 Revisado: Octubre 2013 Aceptado: Diciembre 10 de 2013
}

Por: Elizabeth Montoya Ochoa, ${ }^{1}$, Erika Acevedo Mejia ${ }^{2}$.

\section{RESUMEN.}

En el artículo, derivado de investigación, se muestran las dificultades identificadas en la ciudadela de la Universidad de Antioquia al momento de construir una definición de ambiente en la cual se incluya al ser humano como parte integral del mismo. Para tal fin se aplicaron dos encuestas, la primera que fue denominada "prueba piloto" sirvió para afinar las preguntas usadas en el campo de la psicología a la hora de abordar la preocupación ambiental, ésta es, la escala de Likert; la segunda, fue la encuesta depurada y aplicada entre empleados, docentes y estudiantes de la ciudad universitaria, que ofreció los elementos necesarios para analizar las representaciones sociales del ambiente que comprenden la valoración de los problemas ambientales, el nivel de implicación personal y la capacidad de acción, permitiendo una lectura desde la perspectiva antropológica de las relaciones entre los humanos y el ambiente en ese contexto social.

\section{PALABRAS CLAVE.}

ambiente, representaciones sociales del ambiente, implicación personal, problemas ambientales.

\begin{abstract}
.
This article, derived from research, shows the difficulties identified in the Citadel of the University of Antioquia when it comes to constructing a definition of the environment in which the human being, as an integral part, must be included. For this purpose, two surveys were applied, the first one which was called "a pilot test" served to refine the questions used in the field of psychology in addressing environmental concerns, this is, the Likert scale; the second one, was a refined survey that was applied among employees, teachers, and students, at the University Campus, which offered the elements needed to analyze the social representations comprising the assessment of environmental problems, the level of personal involvement, and the capacity of action, allowing a reading from the anthropological perspective of relationships between humans and the environment in this social context.
\end{abstract}

\section{KEY WORDS.}

Environment; Social Representations of the Environment; Personal Involvement; and Environmental Problems.

\footnotetext{
${ }^{1}$ Antropóloga, Universidad de Antioquia, Medellin. Colombia. elizabethmo.antropologa@gmail.com.

${ }^{2}$ Magister en antropología, Universidad de Antioquia. Medellín. Colombia. erikacevedo85@gmail.com.
} 


\section{Introducción.}

Este artículo aborda la preocupación ambiental entre empleados, docentes y estudiantes en la ciudadela de la Universidad de Antioquia mediante la identificación de las representaciones sociales construidas en este contexto con relación al ambiente. Además del abordaje de las representaciones sociales, se ha evaluado el grado de implicación personal, así como la capacidad de acción prevalente en relación con los problemas ambientales entre los actores mencionados, recurriendo a las metodologias ofrecidas para tal fin por la antropología cognitiva y por la psicologia ambiental.

Se recurrió a la escala de Likert, usada en investigaciones que evalúan las actitudes y opiniones de las personas en un contexto determinado para llevar a cabo la calificación de manera ordinal de una serie de frases elegidas por las investigadoras, con base en una prueba piloto, entre las que el entrevistado determinó su nivel de acuerdo o de desacuerdo. (García, Aguilera, \& Castillo, 2008). Al evaluar estos tres aspectos, las representaciones sociales, el grado de implicación personal y la capacidad de acción, se ofrece la posibilidad de abarcar los elementos fundamentales de la preocupación ambiental entre la población de la ciudadela universitaria, con el objeto de proporcionar herramientas que a futuro permitan solventar esa preocupación, generando posibilidades para la acción.

La investigación fue de tipo analítica, en tanto buscó contrastar y/o comparar los aspectos mencionados en las diferentes categorias de miembros que hacen parte de la comunidad universitaria. Se aplicó un muestreo por conglomerado y asignación proporcional ${ }^{1}$ para cada una de las categorias nombradas, llegando a obtener un total de 1.297 cuestionarios diligenciados. Tomando como punto de referencia que para el momento de la aplicación del cuestionario la población universitaria estaba compuesta por 27.071 personas $^{2}$, repartidas aproximadamente en 1.079 docentes, 24.500 estudiantes y 1.492 empleados (Universidad de Antioquia, 2013), el porcentaje de participación de las personas encuestadas, por categoría de actor, se distribuyó de la siguiente manera: los docentes participaron en un 17\%, los estudiantes en un $4 \%$ y los empleados en un $6 \%$.

\section{Marco teórico}

La preocupación ambiental hace referencia a la actitud de carácter general que tienen las personas hacia el ambiente, además de considerar el conjunto de orientaciones varias que éstas tienen frente a los diferentes temas o acontecimientos medioambientales (González, 2004). A continuación se ofrece una síntesis sobre las perspectivas adoptadas por diferentes autores sobre la preocupación ambiental que permiten tener una claridad conceptual sobre este tema: 


\section{La preocupación ambiental: principales autores y perspectivas}

(Construcción propia, con base en González, 2004)

\begin{tabular}{|c|c|}
\hline $\begin{array}{l}\text { Van Liere y } \\
\text { Dunlap (1981) }\end{array}$ & $\begin{array}{l}\text { Proponen que el concepto de preocupación ambiental está definido en tres temas } \\
\text { fundamentales: la contaminación, la conservación de los recursos naturales y la } \\
\text { regulación del ambiente. }\end{array}$ \\
\hline $\begin{array}{l}\text { Samdahl y } \\
\text { Robertson } \\
\text { (1989) }\end{array}$ & $\begin{array}{l}\text { Plantean tres dimensiones concretas que incluyen aspectos conductuales, como } \\
\text { son: la percepción de los problemas medioambientales, la implicación en } \\
\text { conductas ecológicas y el apoyo a la regulación ambiental. }\end{array}$ \\
\hline $\begin{array}{l}\text { Corraliza y } \\
\text { Berenguer } \\
(1998)-(2000)\end{array}$ & $\begin{array}{l}\text { Determinan una serie de factores que evidencia la preocupación ambiental, tales } \\
\text { como la alarma por las consecuencias o por la gravedad de los problemas } \\
\text { ambientales; el control doméstico relativo a los comportamientos de consumo } \\
\text { energético; la constitución de normas relacionadas con el ambiente; la } \\
\text { información o conocimiento sobre el ambiente; y el locus de control }{ }^{\mathrm{i}} \text { o papel } \\
\text { personal en la crisis energética y ambiental. }\end{array}$ \\
\hline $\begin{array}{l}\text { Corraliza y } \\
\text { Berenguer } \\
(1998)\end{array}$ & $\begin{array}{l}\text { Exponen dos aproximaciones para el estudio de la preocupación ambiental, una } \\
\text { sociológica/antropológica en la que la actitud ambiental es considerada como el } \\
\text { reflejo de los valores y de las creencias socioculturales, y otra de análisis } \\
\text { psicológico o psicométrico, en la que la actitud ambiental es considerada el } \\
\text { reflejo de las dimensiones psicológicas individuales. }\end{array}$ \\
\hline $\begin{array}{l}\text { Aragonés } \\
\text { (1997) }\end{array}$ & $\begin{array}{l}\text { Explica cómo las ciencias sociales abordan el tema de la preocupación ambiental } \\
\text { desde el concepto de "desarrollo sostenible", haciendo alusión a un nuevo } \\
\text { paradigma ambiental como alternativa para entender las relaciones entre el ser } \\
\text { humano y el ambiente, trayendo a colación el carácter ideológico que envuelve } \\
\text { al tema ambiental y apartándolo del carácter meramente evaluativo. }\end{array}$ \\
\hline Schultz (2001) & $\begin{array}{l}\text { Presenta una serie de estudios transculturales que aportan a la comprensión de } \\
\text { las actitudes ambientales individuales. }\end{array}$ \\
\hline
\end{tabular}

Las más recientes aproximaciones a la definición de ambiente ponen en evidencia que uno de los principales desafíos teóricos en torno a esta noción radica en la dificultad para entender las interconexiones e interacciones existentes entre lo natural y humano, "es decir entre todos los componentes de la naturaleza" (Gazzano \& Achkar, 2013: 11). Esa demarcación de los límites en función de las consecuencias ambientales generadas por la acción humana, parte de una perspectiva antropocentrista que posiciona a los humanos por fuera de la naturaleza llevando a la cimentación, desde la psicología, de la noción de preocupación ambiental.

La paradigmática concepción de la ciencia occidental a partir de la cual se hace necesaria la escisión y la construcción de límites materiales e imaginarios entre lo humano y lo no humano, entre lo individual y lo colectivo, entre sujeto y objeto, y entre razón y emoción, ha llegado a constituirse en un limitante para analizar y entender la naturaleza de la preocupación en torno a los problemas ambientales a los que nos vemos enfrentados, como son las altas cantidades de 
emisiones de $\mathrm{CO} 2$ a la atmósfera y el deshielo de los polos para hablar a escala mundial; o los elevados niveles de ruido, los altos consumos de energía y el manejo de los residuos sólidos para ajustar el tema a las problemáticas más presentes en las ciudades. Esta investigación pretende superar ese bache epistemológico que imposibilita, en un contexto determinado, la creación de acciones conjuntas para trascender las preocupaciones por el tema ambiental y llegar a modificar los patrones de conducta individual o colectiva que lleven a la transformación de las actitudes, mediante la comprensión del ambiente como una "totalidad compleja, diversa, en permanente transformación y auto organización, cuya configuración surge de la interacción de procesos físicos, químicos, biológicos, tecnológicos, socio-económicos, políticos y culturales" (Gazzano y Achkar, 2013: 13).

Desde la perspectiva antropológica, el interés por las interacciones entre los humanos y el ambiente viene presentando diferente matices a lo largo de la historia disciplinar. Las interpretaciones realizadas por los diversos grupos sociales del entorno habitado, han sido parte constitutiva del ejercicio de los antropólogos entre las comunidades abordadas. Sin embargo, ante el aumento de las redes de comunicación y el dinamismo económico existente en las relaciones del mundo actual, que hemos denominado globalización, ha sido cada vez más dificil el establecimiento de límites que permitan la comprensión de las actitudes ambientales de las personas como vinculantes a las prácticas de un grupo social específico; es decir, las barreras culturales son cada vez más difusas y en ese sentido las representaciones existentes sobre el ambiente son al mismo tiempo diversas y complejas.

La ciudad universitaria alberga estudiantes provenientes de todas partes del país y esto la constituye en el espacio por excelencia para el abordaje de las diversas aprehensiones existentes del ambiente. Puede decirse que a pesar de carecer de datos etnográficos, la investigación que presentamos se corresponde con la metodología propuesta desde la antropología cognitiva, que tuvo sus origenes en la escuela norteamericana, cuya técnica de análisis de las concepciones de ambiente consistía en formular las mismas preguntas, en el mismo orden, a un cierto número de personas distintas; de esta manera, el analista podía hacerse una idea del conocimiento compartido que la comunidad tenía de su propio entorno (Milton, 1997).

Siguiendo este método, Ellen (1996) identificó tres formas diferentes de aprehender el ambiente. El primero de ellos se caracteriza por abordarlo como "todo aquél espacio que no es humano"; la segunda forma de entender el ambiente es como "una categoria de objetos", en la que aquellos que resultan excluidos de los productos humanos son vistos desde una perspectiva binaria, haciendo una clara distinción entre lo que es natural y lo que es cultural; la tercera forma de interpretar el ambiente es como "una esencia interior" que puede aplicarse tanto al concepto de "ambiente humano", como al ambiente de los seres no humanos. De este modo puede decirse que el concepto occidental de ambiente es polifacético y ambiguo y que dentro de las interpretaciones existentes sobre el mismo, no siempre se halla en el lado opuesto al de la cultura. 
Los antropólogos parecen coincidir en que el modo de comprender el ambiente se deriva de la forma en que las personas lo usan y además de cómo viven inmersos en él. Las amenazas ambientales para la salud humana y las amenazas humanas hacia el entorno constituyen una preocupación de primer orden en todo el mundo. El problema de cómo vivir de un modo sostenible sobre el planeta es generalmente considerado como uno de los retos más severos que debemos afrontar. Dado que sabemos que las actividades humanas son la causa de muchos problemas ambientales, podemos esperar que surja una disciplina académica aplicada a las sociedades humanas y a las culturas, centrada en concreto, en torno a este debate. El debate público sobre el ambiente gira esencialmente alrededor del impacto que las actividades humanas producen sobre su entorno y cómo este impacto puede modificarse para hacerlo menos perjudicial y más tolerable. En este contexto es importante saber no sólo qué tipos de actividad humana perjudican el entorno y de qué modo lo hacen, sino también qué tipos de actividades son inocuas e incluso beneficiosas.

Los biólogos arguyen que la diversidad es la clave de la supervivencia y que una de las principales razones para conservar la biodiversidad es la de hacer probable que la pluralidad de organismos sobreviva y se adapte ante condiciones ambientales adversas. Si la cultura es el mecanismo a través del cual los seres humanos interaccionan con sus entornos, entonces la supervivencia humana puede depender en último extremo de la diversidad cultural. (Milton, 1997).

\section{Metodologia.}

El estudio se realizó con una muestra obtenida de forma aleatoria, mediante un cuestionario aplicado en la plataforma virtual de la universidad, llegando a recaudar 1297 encuestas diligenciadas. La pesquisa fue realizada con base en la información suministrada por estas personas y se llevó a cabo en dos etapas: la primera etapa consistió en el análisis de referentes documentales que permitieron consolidar los parámetros básicos para el establecimiento de las categorias a analizar. Posteriormente se aplicó una prueba piloto que consistió en el envío electrónico de 100 encuestas en formato excel a personas vinculadas con la universidad, las cuales fueron clasificadas de acuerdo con el rol del entrevistado -o por categoría de actor- en la institución, bien fuera como docente, estudiante o empleado.

Se elaboró un instrumento de evaluación de las representaciones sociales del ambiente en la ciudadela universitaria utilizando la escala de Likert , (estableciéndose una media teórica, en rango de 1 a 5, de 2.5). El instrumento que constaba de preguntas cerradas se utilizó en la construcción de categorías con frases de tipo descriptivo, presentando dos enunciados negativos y dos positivos (Garcia, Aguilera, \& Castillo, 2008). Ésto con la finalidad de identificar y de evaluar el nivel de preocupación ambiental mediante un análisis estadístico que determinara las categorias a utilizar en la evaluación de las representaciones sociales, la valoración de los problemas ambientales, los niveles de implicación personal en la comunidad universitaria y la capacidad de acción.

En total se recibieron 34 encuestas diligenciadas luego de la prueba piloto, a través de las cuales los participantes fueron incentivados a realizar comentarios, sugerencias y a exponer el grado de ambigüedad encontrado en las preguntas 
según sus concepciones de ambiente, medio ambiente, territorio e identidad - Lo anterior permitió hacer el cotejo correspondiente a las respuestas para la posterior revisión y reformulación de las preguntas teniendo en cuenta las dificultades expresadas por los encuestados ante los cuestionamientos iniciales.

La segunda etapa consistió en el diseño de la encuesta final, construida con base en los resultados de la prueba en mención a partir de la cual, previo establecimiento de las variables dependientes teóricas, se formularon y reformularon los cuestionamientos que serían aplicados en el curso de la investigación. Para el análisis de las respuestas obtenidas mediante la aplicación de la encuesta, se acudió a las herramientas teóricas y conceptuales de la psicología ambiental y de la antropología cognitiva.

\section{Resultados - discusiones.}

Las variables teóricas tenidas en cuenta para la evaluación de la preocupación ambiental fueron: la representación social del ambiente (en adelante RSA) que hace mención a un sistema de valores que va proporcionando a las personas mecanismos de orientación en su contexto, material o social, y que a su vez permite compartir modelos generalizados colectivos que son interiorizados por medio de los canales de comunicación (Gutiérrez 1998). Además la RSA comprende a) la valoración de los problemas ambientales en general (en adelante VPA); b) la implicación personal (en adelante IP) que permite conocer qué tan dispuestos están los encuestados a involucrarse ya sea en un tema, o en una acción, lo cual da cuenta de la implicación comportamental, o de la motivación que tiene la persona o el grupo al que pertenece para llevar a cabo acciones pro-ambientales ; y c) la capacidad de acción (en adelante CA) ante los problemas ambientales (Black, Stern y Elworth, 1985 en Berenguer y Corraliza, 2000) que se refiere a la facultad que tienen las personas para hacer las cosas en orden de importancia, y no en las intenciones que éstas puedan tener en un hecho concreto (Long, 2007).

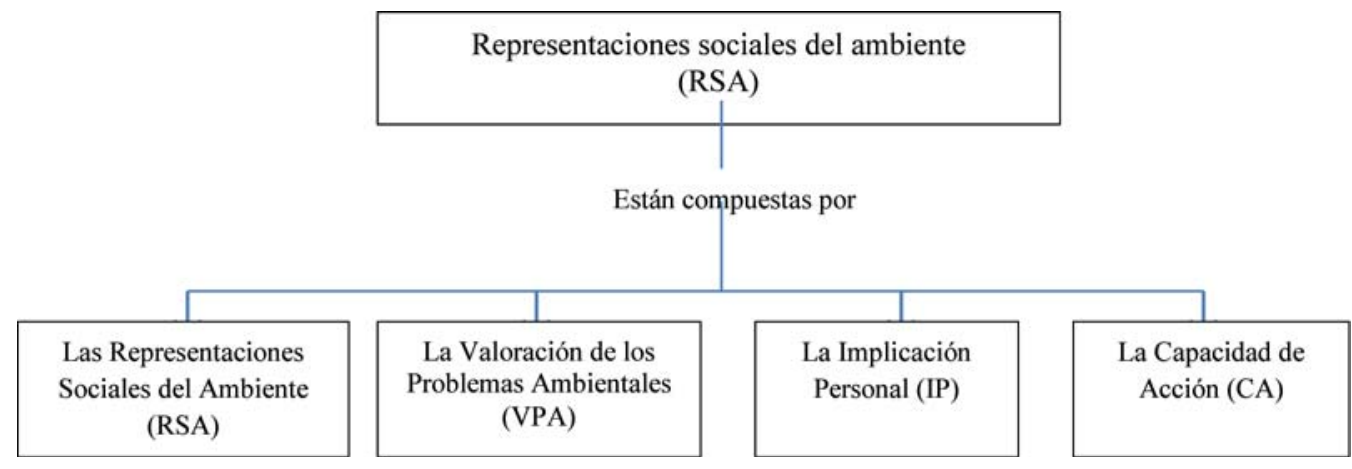

(Construcción propia con base a las teorías revisadas)

Representaciones sociales del ambiente

Esta variable, comprende el análisis de la valoración de los problemas ambientales en la comunidad universitaria. Tuvo como fuente de valoración las ideas que las personas tienen acerca de los árboles, los animales, la contaminación, el cuidado, la tranquilidad, el aire, la naturaleza, las enfermedades, el agua y la salud, que 
durante la prueba piloto caracterizaron siempre y en todos los casos la concepción de ambiente entre la población abordada.

Las representaciones sociales pueden definirse como un proceso cognitivo que tienen una fuerte incidencia en la construcción social del conocimiento, en tanto poseen unos contenidos, instrumentos o actitudes, que se estructuran formando conjuntos organizados en reglas de funcionamiento social (Navarro, 2004). Esas actitudes son definidas como el conjunto de creencias, sentimientos y tendencias psicológicas de un individuo que se expresan mediante la evaluación de una entidad $\mathrm{u}$ objeto, en este caso el ambiente, dando lugar en ocasiones a un comportamiento determinado por esa actitud (Ospina, et al.: 2005). En ese orden de ideas, los comportamientos colectivos obedecen a un conjunto de actitudes que han sido naturalizadas o normalizadas bajo el rasero de las representaciones colectivas.

La RSA y el sistema de valores que la conforman ofrecen los medios necesarios para la orientación de los mecanismos de adaptación de las personas a su condición social, histórica y física (Silva, 2002). Es así como estas RSA se construyen simbólicamente en el marco de las interacciones sociales, dándole sentido a la realidad, creando imaginarios, comportamientos, demarcando y conceptualizando lo real con respecto a lo conocido. Su dinamismo genera formas de entendimiento, comunicando las realidades mediante diálogos constantes con las prácticas cotidianas (Ocaña y LópezHernández, 2005: 2). Los resultados obtenidos de la evaluación de este indicador entre la población de la ciudad universitaria, dejan claro que la definición de ambiente que poseen los entrevistados es diversa y variable.

\section{Gráfica I: RSA}

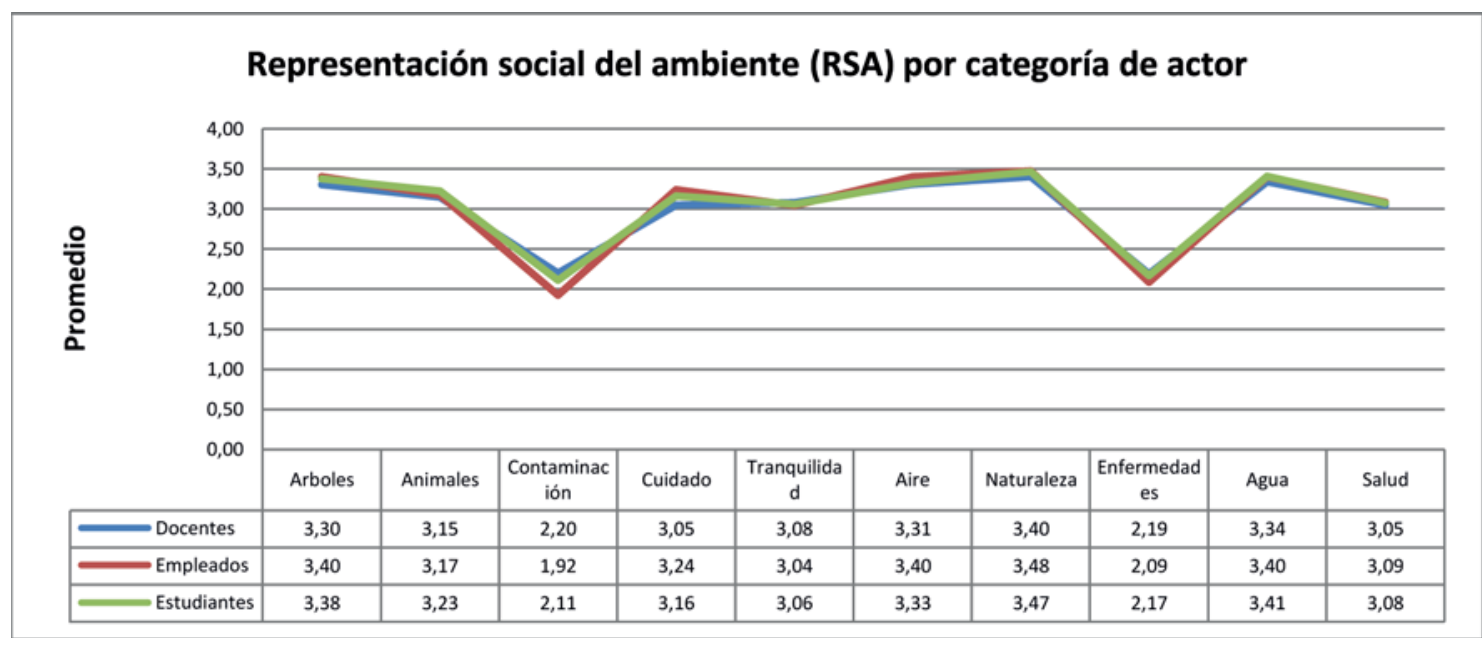

A partir de los resultados entendemos que en la categoría de actor, de la Gráfica I, para los habitantes del campus universitario la RSA, la contaminación y las enfermedades son elementos periféricos al ambiente natural, para los empleados la contaminación está dada por debajo de la media teórica de (2.5), seguido muy de cerca de los estudiantes. Para el nivel de profesores tiende a subir un poco aunque sigue estando por debajo de la media teórica. Las enfermedades se encuentran en el tope de la media teórica (2.5), de tal forma que estas últimas caracterizan 
siempre y en todos los casos al ambiente, pero dejándolo por fuera del ambiente central, ambiente natural, y no construido (U1loa, 2001).

De acuerdo con la gráfica anterior el cuidado del ambiente contiene múltiples dimensiones, y posee un nexo demostrado con problemas que dentro del imaginario de las personas no se conciben como asuntos relacionados con el ambiente. La representación social que se tiene en la comunidad de la ciudad universitaria frente al cuidado y la protección ambiental se relaciona directamente con las condiciones histórico-sociales e ideológicas determinadas por la memoria colectiva y el sistema de normas que se instauran en la institución.

La percepción estaría compuesta entonces por el conocimiento, las ideas, sentimientos, emociones y opiniones que las personas tienen de una situación (Aragonés y Jiménez, 1991). Por consiguiente, el mecanismo utilizado en este trabajo para analizar la percepción que las personas tienen del ambiente sirvió, en última instancia, para llegar a identificar y comprender cuáles son los principales elementos que preocupan a los docentes, estudiantes y empleados del campus universitario, en relación con el tema ambiental. El indagar por los conocimientos que tienen las personas en torno al tema, es decir, qué piensan y qué sienten frente a una problemática determinada, permitió identificar la existencia o no de una preocupación ambiental en el contexto universitario, además de determinar las representaciones sociales del ambiente.

Con base en lo anterior, puede afirmarse que la RSA, permitió valorar los problemas ambientales en general y los problemas ambientales de la comunidad universitaria en particular, encontrando, de acuerdo con la media teórica establecida 2.5, que las personas tienen un sentido periférico frente a los temas de contaminación, en temas relacionados con los ítems la salud y las enfermedades; es decir, que no lo relacionan con lo ambiental. Lo contrario ocurre con items como la naturaleza, el agua, el aire, los árboles, los animales, la salud, la tranquilidad y el cuidado, a los cuales se les otorga un carácter central, lo que sugiere que la RSA, se corresponde con el imaginario de un ambiente "natural", "verde", que genera salud y tranquilidad. Es legitimo pensar igualmente que las RSA, constituyen una especie de telón de fondo de las representaciones sociales de otros objetos ambientales, como por ejemplo los riesgos ambientales o incluso de la calidad ambiental.

Lo anterior deja entrever que para los habitantes del campus universitario el ambiente está basado en lo natural, la gran mayoría lo consideran como algo externo, fuera de ellos, y no lo relaciona con su individualidad, lo que implica que el cuidado se delega a los demás, al Estado, a los Directivos de la Universidad, a las Corporaciones Ambientales Colombianas, en fin, a otros actores diferentes a ellos y no como un acto individual que requiere del compromiso y del esfuerzo colectivo para lograr la construcción de una conciencia ambiental.

La valoración de problemas ambientales.

La valoración de los problemas ambientales sugiere que aunque existen elevados niveles de preocupación ambiental, no es posible determinar claramente si las personas de la comunidad universitaria presentan una estimación de los problemas ambientales fundamentados en los conocimientos ofrecidos por los currículos de la universidad. La necesidad principal que se manifiesta a través de esta investigación es que la Institución 
debe visibilizar sus acciones frente al cuidado y la protección ambiental, incorporando currículos académicos transversales a todas las áreas del saber.

Es fundamental que al interior de la institución universitaria se considere la posibilidad de estimular la reformulación de las políticas ambientales existentes, además de facilitar la implementación de las políticas que desde la Constitución Politica de Colombia de 1991 pretenden garantizar un ambiente protegido ${ }^{4}$; lo anterior, sin ser ajenos a los problemas políticos que limitan las funciones de las autoridades ambientales, encargados por las leyes del Estado Colombiano, y en consecuencia por los ciudadanos en el establecimiento de planes ambientales efectivos y duraderos.

Podemos entonces afirmar que, evaluar el nivel y las características de la preocupación por la calidad ambiental que tienen las personas que hacen parte de la comunidad universitaria (docentes, estudiantes, empleados), a través del reconocimiento de sus representaciones, valoraciones y prácticas con respecto a su entorno universitario demuestra, en general, que los problemas ambientales están dados básicamente en torno a las representaciones sociales que tienen introyectadas cada una de las personas en su referente de vida y que es determinado por las relaciones sociales que establecen a lo largo del tiempo.

Por último, las dimensiones utilizadas en este estudio que constituyen (o por lo menos describen) la preocupación ambiental en la comunidad universitaria de la Universidad de Antioquia, probaron ser pertinentes para exponer la complejidad del objeto de estudio en cuanto a las relaciones teóricas propuestas desde la psicología social ambiental. Como lo establece Moser (2003), éstas permitieron reconocer los métodos que hacen posible la relación de las personas con el ambiente, sus percepciones, actitudes, las evaluaciones de sus representaciones sociales sobre los comportamientos y conductas ambientales característicos en las personas.

Antropológicamente esta investigación permitió incorporar la relación de fenómenos sociales frente a los problemas ambientales (Ulloa, 2001), relacionando la persona con el ambiente, partiendo desde la representación social.

Implicación personal.

Como ya se explicaba, la preocupación ambiental en esta investigación fue valorada mediante el hallazgo de los promedios y el análisis estadístico de los datos recaudados en la encuesta, construida con base en las lecturas previas y en la prueba piloto. Los resultados obtenidos fueron codificados otorgándoles un valor desde uno (1) que significa que la persona está totalmente en desacuerdo, hasta cuatro (4) que significa que está totalmente de acuerdo; otras preguntas cuestionaban la facultad que tienen las personas para hacer las cosas en orden de importancia, y no en las intenciones que éstas puedan tener en un hecho concreto (Long, 2007). Dentro de las escala de valor asignada, el uno (1) significaba que la persona no ofrecía ninguna importancia al tema tratado; mientras que cuatro (4) fue la calificación estipulada a quienes consideraban que el tema tratado era muy importante. Otras preguntas tenían una escala de calificación, de uno a cuatro, en la que uno (1) significaba que nunca ejecutaba prácticas pro-ambientales y cuatro (4) significaba que la persona siempre estaba dispuesta a ejecutar este tipo de prácticas. 
Los problemas ambientales generan interés debido a que éstos pueden fomentar mecanismos para prever, además del comportamiento ecológico, las actitudes y creencias pro-ambientales (Black, Stern y Elworth, 1985 en Berenguer y Corraliza, 2000:325). Para hacer esta valoración se tomaron las siguientes dimensiones:

- Identificación: Los problemas ambientales me importan más que cualquier cosa; me siento comprometido; me siento afectado.

- Valoración: Los problemas ambientales deberian suscitar interés en la mayoria de las personas; son un tema preponderante en la sociedad; tienen un peso altamente considerable.

- Percepción de la Posibilidad de Acción: Estoy en capacidad de actuar para solucionar los problemas ambientales; mis capacidades para actuar son muy importantes; involucrarse en los problemas ambientales permite cambiar estos problemas.

Gráfica II: IP

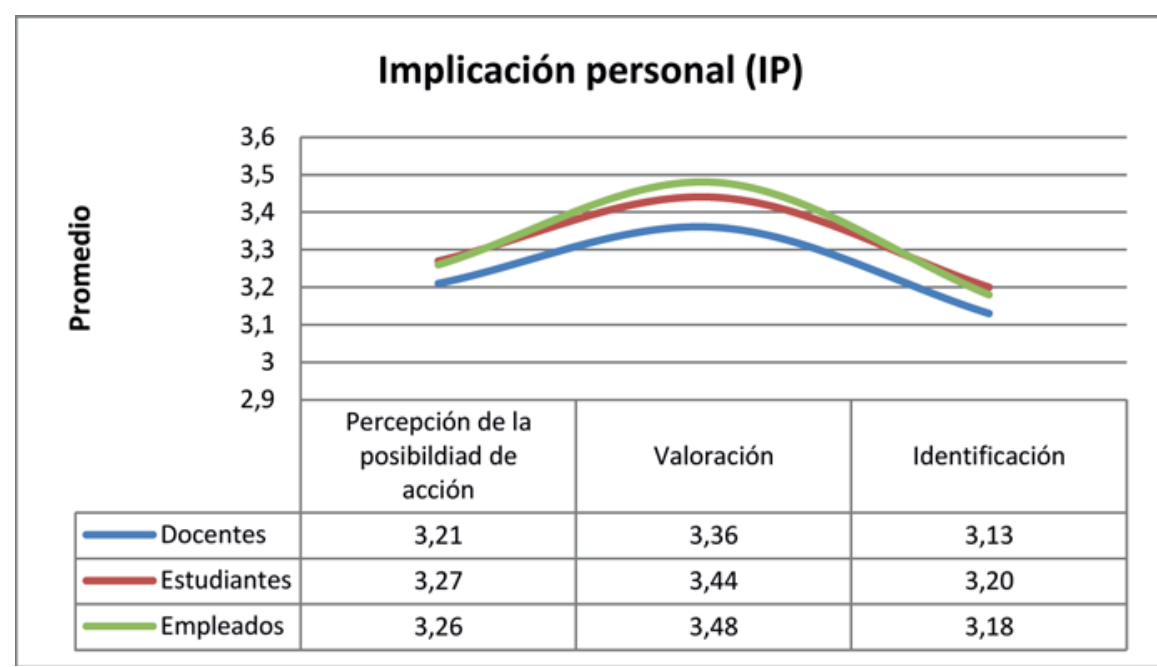

Como se puede apreciar en la Gráfica II, la implicación personal plantea que en el aspecto de la identificación los estudiantes presentan un promedio $(3,20)$ superior a los empleados $(3,18)$ y los docentes $(3.13)$, esta situación es consecuente con la necesidad que tienen los estudiantes de pertenecer a un lugar, de tener identidad, mantener el soporte local, conservar la familiaridad, en resumen, de generar redes sociales en el espacio habitado, el campus universitario; apropiándose de las relaciones sociales, comportamientos, cogniciones y sentimientos.

Respecto a la valoración de los problemas ambientales, para los empleados $(3,48)$ se encuentra por encima de la valoración realizada por los estudiantes $(3,44)$ y los docentes $(3,36)$, lo que evidencia, según Black et al (1985), que las personas hacen una valoración correspondiente a la problemática ambiental otorgando importancia al compromiso, a las relaciones de pautas del comportamiento humano y de la organización social en la que se involucran las actitudes ambientales. En cuanto a la percepción de la posibilidad de acción se presenta en un promedio similar para los estudiantes $(3,26)$ y los empleados $(3,27)$; mientras que para los docentes $(3,21)$, si bien la cifra no es baja, sí lo está comparativamente con los dos anteriores. De esto 
se deduce que la capacidad de actuar en la solución de los problemas ambientales es más factible en los empleados y los estudiantes que en los docentes.

Lo anterior permite concluir que existen niveles elevados de preocupación ambiental, pero no se puede determinar claramente si las personas de la comunidad universitaria tienen una valoración de los problemas ambientales basados en las creencias y valores ambientales que puedan llegar a definir una condición ajustada a la problemática ambiental del campus universitario.

Capacidad de Acción.

Para la antropología la CA es una postura trabajada bajo la denominación de "capacidad de agencia", para Long (2007) esta capacidad de agencia permite procesar la experiencia, diseñando formas para enfrentarse a situaciones bajo los parámetros más dificiles o más sencillos, pero siempre manteniendo claro el límite, la incertidumbre y las demás restricciones que el entorno pueda ofrecer. Esta se desarrolla como un indicador para el pronóstico de la posibilidad de acción y de la pertinencia y eficacia de la misma; además, permite evidenciar la predisposición del sujeto para la acción, que es juzgada y que consta de la dimensión de (Flament y Rouquette, 2003) Identidad Social, la cual es definida por el grado de identificación de la persona a propósito del objeto.

Según esto, en la CA los Estudiantes y los Empleados están más implicados en los problemas ambientales, no obstante al observar las tres categorias de actores se hace evidente que el tema de la suciedad adquiere una importancia fundamental, lo que hace posible pensar en que este item en particular se encuentra vinculado socialmente al concepto de contaminación. Para este caso en particular, la contaminación se convierte en una representación social del objeto ambiental (Ferreira, 2002), dado que las personas se adaptan al contexto y a las condiciones físicas del ambiente construyendo unos patrones estéticos que en esta ocasión están asociados para los entrevistados con los espacios limpios.

\section{Conclusiones.}

Mediante esta investigación pudo identificarse la asociación directa que realizan los encuestados entre ambiente construido, que equivaldría a lo que conocemos como las zonas que cuentan con infraestructura lograda por humanos y ambiente natural que sería aquél que carece de objetos construidos por humanos ${ }^{5}$. De esta manera en la ciudad universitaria, los árboles, por ejemplo, se consideran parte del ambiente natural a pesar de encontrarse en un contexto que las personas normalmente identificarian como ambiente construido.

Para abordar la preocupación ambiental, es importante entender las representaciones que las personas tienen del ambiente y, por supuesto, las congruencias que éstas poseen con eluso social del territorio, entendiendo que ese territorio no puede tomarse en abstracto y que se concreta en dominios culturales como son el parentesco, la religión, la economía y en nuestro caso, la academia (Nates, 2011). Debe resaltarse que entre las diversas representaciones de ambiente halladas en las encuestas, existe una en particular que consiste en la homologación, en términos cognitivos, de las nociones de naturaleza y territorio, aspecto preponderante dado que la Unidad 
de Coordinación de Educación Ambiental de la República de Argentina considera que ésta es una concepción fundamental porque permite visualizar "la naturaleza como territorio, como lugar para vivir acorde un proyecto cultural comunitario" (2009: 149). Probablemente en esa homologación de conceptos y en su aplicación a los diversos dominios de la vida cotidiana de las personas pueda estar la clave para superar la barrera de la preocupación, logrando convertirla en acción socioambiental.

La valoración de los problemas ambientales entre la población de la ciudad universitaria muestra la existencia de altos niveles de preocupación ambiental $(3,43)$, mientras que el nivel de identificación social tuvo un promedio más bajo $(3,19)$ lo cual muy probablemente se deba a las múltiples representaciones, que los diversos sujetos que habitan en la ciudad universitaria, poseen del ambiente. Sin embargo, la posibilidad de acción percibida al interior del grupo total de entrevistados $(3,25)$ hace alusión a que dentro del campus universitario, con las medidas adecuadas en términos de educación en materia ambiental, las personas estarian dispuestas a realizar acciones pro-ambientales sin que esto represente mayor esfuerzo para los estamentos de la institución. Además, pudo identificarse que la ciudad universitaria presenta una problemática en el campo de la identificación social de tal magnitud que reduce la percepción de la posibilidad de acción individual, lo cual sin lugar a dudas afecta la posibilidad de acción colectiva.

El respeto por la diversidad cultural y biológica, como observamos en la perspectiva antropológica, puede ser un elemento constitutivo de una nueva relación entre el ambiente y sociedad. La perspectiva antropológica puede ofrecer los medios para ejemplificar diversos modos de relación y de concepción del ambiente en escenarios etnográficos diferentes, llegando a aportar un panorama amplio de casos que han logrado solventar problemas históricos en las relaciones humanos/ambiente. Queda pendiente entonces la contextualización histórica y etnográfica de las personas abordadas en las encuestas y la aplicación de períodos de observación directa de las acciones de los entrevistados.

Consideramos finalmente que el empoderamiento por parte de la población universitaria de los conocimientos necesarios para solventar las problemáticas ambientales actuales, puede tener un respaldo en las aulas de clase a través de la comprensión en términos científicos de esas problemáticas en la relación humano/ ambiente. Argüimos que el proceso de formación en temas ambientales pude llegar a constituir un cambio en las representaciones del ambiente en la ciudad universitaria, logrando una trascendencia de la preocupación, hacia la actuación. 


\section{Referencias bibliográficas.}

Aguilar Luzón, M. (2006) Predicción de la conducta de reciclaje a partir de la teoria de la conducta planificada y desde el modelo del valor, normas y creencias hacia el medio ambiente. Tesis doctoral. Universidad de Granada. Recuperado el 27 de mayo de 2014, de http://0-hera.ugr.es.adrastea.ugr.es/tesisugr/16135593. pdf.

Aragonés, Juan y Jiménez Burillo, Florencio (1991). Introducción a la psicología ambiental. Vol. 15. Madrid: Ed. alianza.

Berenguer, J \& Corraliza, J. (2000). Preocupación ambiental y comportamientos ecológicos [Versión electrónica], Psicothema 12, (3), 325-329. Recuperado el 13 de noviembre de 2012, de www.unioviedo.net/reunido/index.php/PST/ article/download/.../7463

Black, J.S., Stern, P.C. \& Elworth, J.T. (1985). Personal and contextual influences on househould energy adaptations. Journal of Applied Psychology 70 (1), 3-21.

Corraliza, J. A. \& Berenguer, J. (1998) Estructura de las Actitudes Ambientales: ¿orientación general o especialización actitudinal? Revista de Psicología Social.13 (3). pp. 399-406.

Dunlap, R., Kent V-L., Mertig, A. \& Emmet J., R. (2000). Measuring endorsement of the new ecological paradigm. A revised NEP scale [Versión electrónica], Journal of Social Issues 56, (3), 425-442. Recuperada el 15 de febrero de 2014 de www.researchgate.net/...New...Measuring...Measurin...

Ellen, Roy (1996) “The cognitive geometry of nature: a contextual approach"' En Gisli, Palsson y Philiphe Descola (eds) Nature and Society: anthropological perspectives. Londres y Nueva York: Routledge.

Ferreira, R. (2002) Representaciones sociales de medio Ambiente y educación ambiental de Docentes universitarios, en: Tópicos en educación ambiental. Vol. 4. $\mathrm{N}^{\circ}$ 10. pp. 22-36.

Flament, C. \& Rouquette, M. (2003). Anatomie des idées ordinaires. Comment étudier les représentations sociales. Paris-Francia: Armand Colin.

García, S. J., Aguilera, J. \& Castillo, A. (2011, enero-junio). Guía técnica para la construcción de escalas de actitud [Versión electrónica], Odiseo Revista Electrónica de Pedagogía, Año 8, (16). Recuperado el 13 de mayo de 2013, de http://www.odiseo.com.mx/articulos/guia-tecnica-para-construccionescalas-actitud.

Gazzano, I. \& Achkar, M. (2013, diciembre). La necesidad de redefinir ambiente en el debate científico actual [Versión electrónica], Revista Gestión y Ambiente 16, (3), 7-15. Recuperado el 3 de enero de 2014, de http:/ / www.revistas.unal. edu.co/index.php/gestion/article/viewFile/38052/44266. 
González López, A. (2002). La preocupación por la calidad del medio ambiente. Un modelo cognitivo sobre la conducta ecológica. Tesis para optar al titulo de Doctor en Psicología social, Universidad Complutense de Madrid. Facultad de Psicología. Madrid-España. Recuperado el 25 de abril de 2014, de http:// biblioteca.ucm.es/tesis/psi/ucm-t26479.pdf.

Gutiérrez Alberoni, J.D. (1998). La teoría de las representaciones sociales y sus implicaciones metodológicas en el ámbito [Versión electrónica], Psiquiatría Pública, 10, (4), 221-219. Recuperado el 25 de junio de 2012, de http:// www.insumisos.com / lecturasinsumisas / Teorias \% 20de\% 201 as $\% 20$ representaciones.pdf.

Long, N. (1997). "Sociología del desarrollo: una perspectiva centrada en el actor". Centro de Investigaciones y Estudios Superiores en Antropología Social: E1 Colegio de San Luis: México. COLSAN, CIESAS.

Milton, K. (1997) Ecologies: anthropology, culture and the environment. Part of: International Social Science Journal. Vol. 49. Nº154. pp. 477-495. UNESCO.

Moser, G. (2003). La Psicología Ambiental en el Siglo XXI [Versión electrónica]. El Desafio del Desarrollo Sustentable 12 (2), 11-17. Recuperado el 21 de marzo de 2013, de http://www.analesderecho.uchile.cl/index.php/RDP/article/ viewFile/17386/18146.

Navarro, O. (2004) Representación social del agua y de sus usos. Psicología desde el Caribe, Núm. 14, diciembre, 2004, pp. 222-236. Universidad del Norte, Barranquilla, Colombia. En internet: http://www.redalyc.org/ pdf/213/21301409.pdf (Acceso: 09/05/2014).

Ocaña, R. Leticia y López-Hernández, Eduardo (2005). Representaciones Sociales de Conservación Ambiental en la comunidad de Flor de Márquez de Comillas, en: Educación Ambiental. Vol. 4. N². pp. 1-11. Universidad Juárez Autónoma de Tabasco: México.

Orejas, A. (1995). Del marco geográfico a la arqueología del paisaje. La aportación de la fotografía aérea. Monografias 15. Madrid-España: Consejo Superior de Investigaciones Científicas (CSIC).

Ospina, B; Juan de Jesús Sandoval; Carlos Andrés Aristizábal Botero; Martha Cecilia Ramírez Gómez (2005) La escala de Likert en la valoración de los conocimientos y las actitudes de los profesionales de enfermería en el cuidado de la salud. Antioquia, 2003. En: Investigación y Educación en Enfermería Medellin, Vol. XXIII N. ${ }^{\circ}$ 1, marzo de 2005. En internet http://www.redalyc. org/articulo.oa?id=105215401002 (Acceso: 09/05/2014).

Samdhal, D. M. \& Robertson, R. (1989) Social Determinants of Environmental Concern. Specification and Test of the Model. Environment and Behaviour, 21. pp. 57-81.

Silva A., L. C. (1993). Muestreo para la investigación en ciencias de la salud. MadridEspaña: Díaz de Santos. 
Ulloa, A. (2001). Transformaciones en las Investigaciones Antropológicas: sobre naturaleza, ecología y medio ambiente, en: Revista Colombiana de Antropología. $N^{\circ} 37$. pp. 188-232.

Universidad de Antioquia, (2013). En internet http://docencia.udea.edu.co/ vicedoce/publicaciones/indicadores. (Acceso: 17/08/2013).

\section{Notas:}

$1 \mathrm{El}$ muestreo por conglomerados es utilizado en poblaciones divididas de forma natural, es decir en grupos que, se supone, abarcan la variabilidad de toda la población representándola rigurosamente con respecto a la característica a elegir, pudiéndose seleccionar sólo algunos de estos grupos o conglomerados para la realización del estudio. De igual manera la asignación proporcional, como su nombre lo indica, permite considerar proporcionalmente el tamaño de la muestra dentro de cada estrato al interior de la población elegida (conglomerado o grupo), (Silva L.C, 1993).

2 Los datos poblaciones la Ciudad Universitaria fluctúan permanentemente. La UdeA por ser una institución pública mantiene un constante movimiento de personal, tanto de empleados, como de docentes y de estudiantes. Consultado el 17 de agosto de 2013. http://docencia.udea.edu.co/ vicedoce/publicaciones/indicadores.

3 El locus de control, también conocido como control interno-externo, es un concepto que describe el hecho de que para una tarea determinada, las personas se comportan de forma muy diferente según crean que su ejecución depende de ellos mismos o no. Las personas que creen que el desarrollo de determinada tarea depende de ellos mismos tienen un locus de control interno, mientras que las convencidas de que el desarrollo exitoso de la tarea depende de factores externos tienen un locus de control externo. "Se considera que el locus de control es un rasgo de la personalidad estable en el tiempo, y sitúa a las personas en un continuo según la responsabilidad que aceptan sobre los eventos sociales que experimentan -que pueden ser positivos, negativos o neutros-. Así se muestra el grado en que un individuo percibe el origen de su propio comportamiento, ya sea de manera interna o externa a él". Consultado el 15 de diciembre de 2013. Consultado en: http://espectroautista.info/ tests/sociabilidad/habilidades-sociales/SOC3.

4 Ver por ejemplo el problema de las basuras en Medellin:

http://www.concejodemedellin.gov.co/concejo/concejo/index.php?sub_cat=9214\#.U3Zj0_15OuI

5 Además, si se observan con detenimiento las dificultades que enfrentan los académicos para abordar el concepto de ambiente, se notará también su influencia en la población secular, la cual ha construido todo su sistema cognitivo tomando elementos impregnados de esas dificultades teóricas.

6 La escala de Likert es usada en la mayoría de las investigaciones que evalúan actitudes y opiniones de las personas en un contexto determinado. En la escala de Likert se califican de manera ordinal una serie de frases elegidas en una escala que determina el nivel de acuerdo o de desacuerdo del entrevistado. Ver: http://www.redalyc.org/articulo.oa?id=105215401002

7 Fue común observar que la mayoría de las personas encuestadas durante la prueba piloto, no encontraban diferencias conceptuales entre las palabras ambiente, medio ambiente, entorno y espacio. Esta homologación dentro del ideario de los entrevistados, nos llevó a comprender la inoperancia de realizar encuestas basadas en los constructos académicos relacionados con los estudios ambientales, llevándonos también a retomar aquellas definiciones coloquiales que se asocian por la mayoría de las personas al concepto de 'medio ambiente' como son: naturaleza, árboles, reciclaje, salud, entre otras.

8 La predicción de las acciones pro-ambientales ha sido abordada desde la psicología social de diversas maneras, algunas de las cuales homologan la conducta ambiental con el comportamiento ambiental, 
aspecto que según muestra Aguilar Luzón (2006) puede resultar problemático, pero cuya discusión no se corresponde con los objetivos de esta investigación. En este escrito entendemos las actitudes proambientales como las acciones sobre el ambiente, motivadas por la percepción subjetiva de riesgo ante una amenaza tal que ponga en peligro la supervivencia de las personas, por ejemplo, el desabastecimiento de agua. Una conducta pro-ambiental en estos términos sería toda aquella acción basada en una reflexión sobre esos riesgos que tendría repercusión directa en la conservación de los recursos en condiciones adecuadas para la supervivencia humana. Esta perspectiva, si bien hace alusión a la dimensión egoísta de las acciones pro-ambientales, también ofrece la posibilidad de entender el proceso reflexivo y de identificación que involucra la relación de los humanos con su ambiente.

9 La protección del ambiente es una obligación constitucional. La Constitución Nacional, en su artículo 79 consagra que: "todas las personas tienen derecho a gozar de un ambiente sano. La Ley garantizará la participación de la comunidad en las decisiones que puedan afectarlo. Es deber del Estado proteger la diversidad e integridad del ambiente, conservar las áreas de especial importancia ecológica y fomentar la educación para el logro de estos fines". Ver: http://www.upme.gov.co/guia_ ambiental/carbon/gestion/politica/normativ/normativ.htm

10 Otros autores se apoyan también en esta definición de ambiente natural; tal es el caso de Orejas, quien considera que ambiente natural "es entendido" como el estadio de equilibrio entre lo abiótico y lo biótico, sin intervención de origen humano (1995: 61). De esta manera, cuando esos dos componentes, el biótico y el abiótico, se ven alterados desaparece el equilibrio. Ejemplo de ello es que la historia de cada unidad de producción -la agricultura, la ganadería, la industrialización, etc. - viene marcada por diversas fases de equilibrio y desequilibrio. El sistema industrial en el que se contextualiza nuestra investigación, se encuentra en una fase de clara inestabilidad, lo cual habla precisamente de esa ruptura del equilibrio del medio natural. 